\title{
Civilisations
}

Revue internationale d'anthropologie et de sciences

humaines

$51 \mid 2004$

Religions transnationales

\section{La transnationalisation du pentecôtisme brésilien : le cas de l'Eglise Universelle du Royaume de Dieu}

\section{Ari Pedro Oro}

\section{OpenEdition}

\section{Journals}

Édition électronique

URL : http://journals.openedition.org/civilisations/683

DOI : $10.4000 /$ civilisations. 683

ISSN : 2032-0442

\section{Éditeur}

Institut de sociologie de l'Université Libre de Bruxelles

\section{Édition imprimée}

Date de publication : 1 janvier 2004

Pagination : 155-170

ISSN : 0009-8140

\section{Référence électronique}

Ari Pedro Oro, « La transnationalisation du pentecôtisme brésilien : le cas de l'Eglise Universelle du Royaume de Dieu », Civilisations [En ligne], 51 | 2004, mis en ligne le 07 janvier 2009, consulté le 23 avril 2019. URL : http://journals.openedition.org/civilisations/683 ; DOI : 10.4000/civilisations.683

Ce document a été généré automatiquement le 23 avril 2019

(C) Tous droits réservés 


\title{
La transnationalisation du pentecôtisme brésilien : le cas de l'Eglise Universelle du Royaume de Dieu
}

\author{
Ari Pedro Oro
}

1 L'Eglise Universelle du Royaume de Dieu (EURD) est l'un des plus impressionnants phénomènes religieux des dernières décennies au Brésil et, dans un certain sens, dans l'ensemble du monde occidental. Fondée en 1977 à Rio de Janeiro, elle jouit aujourd'hui d'une grande visibilité, bien que, selon le dernier recensement national de l'année 2000, elle occupe la troisième place parmi les Eglises évangéliques brésiliennes, avec ses 2 millions de fidèles, ses 7.000 temples et ses 14.000 pasteurs $^{1}$. Dans ce pays, elle est considérée comme un véritable empire économique et médiatique, et détient un réel pouvoir politique. C'est aussi l'Eglise du Sud qui, de toutes les Eglises évangéliques, rencontre le plus de succès dans son expansion transnationale, puisqu'elle a implanté des temples dans quatre-vingts pays des quatre continents. Il semble bien que pour cette Eglise, le terme » universel soit plus qu'un nom; c'est une idée, une ambition» (Ruuth et Rodrigues, $2000: 29$ ).

2 Le présent article traite de cette dimension transnationale de l'Eglise Universelle. Evidemment, d'autres Eglises pentecôtistes brésiliennes ont traversé les frontières nationales. Tel est le cas de Dieu est Amour, Renaître en Christ et Assemblée de Dieu. Néanmoins, celles-ci sont loin d'avoir la même importance que l'EURD, même si l'Eglise Universelle est aujourd'hui une des nombreuses » méga-Eglises » à caractère mondial. Comme nous avons écrit ailleurs :

"Les plus connues des méga-Eglises proviennent des pays du Nord. Il s'agit en particulier des Assemblies of God qui seraient présentes, selon leurs propres informations, dans 186 pays et atteindraient 35 millions de personnes. Les autres Eglises comme la Church of God, la Church of God of Profecy, la Four-square Gospel Church, la Church of God in Christ, la Rhema Church ou l'International United Pentecostal Church ont des prétentions moindres, mais revendiquent parfois jusqu'à 10 millions de personnes. En dehors de ces Eglises émanant des Etats-Unis et de quelques autres très puissantes émanant de 
Grande-Bretagne (Apostolic Church) ou de Scandinavie (Eglise de la Pentecôte), il faut souligner la croissance récente d'Eglises multinationales provenant de pays du Sud. Elles sont issues de Porto Rico, du Mexique, d'Argentine, du Brésil, de Bolivie, du Nigeria, du Ghana, d'Afrique du Sud, de Corée, etc. » (Oro, Corten et Dozon, 2003 : 24)

3 En ce qui concerne la dimension transnationale de l'Eglise Universelle, nous nous attachons principalement à analyser, tel que le préconise R. Segato pour les études sur la transnationalisation religieuse, la manière dont elle se localise, s'« indigénise ». A partir de l'analyse de l'implantation de l'EURD en Argentine, au Portugal et en Afrique du Sud, nous verrons comment cette Eglise a développé une capacité toute particulière à s'adapter aux contextes culturels locaux ${ }^{2}$. C'est pourquoi, comme le soutient aussi R. Segato (1997), l'interprétation du succès de la transnationalisation de l'Eglise Universelle ne peut pas laisser de côté la référence à la nation. Mais, avant d'aborder la question de la transnationalisation de l'Eglise Universelle, nous allons analyser son succès sous trois angles : médiatique, économique et politique.

\section{Les différentes facettes du succès de l'Église Universelle du Royaume de Dieu}

4 Le fondateur de l'Eglise Universelle du Royaume de Dieu est le carioca ${ }^{3}$ Edir Macedo, cinquante-sept ans, ancien employé de la loterie de l'Etat de Rio de Janeiro qui est passé par le catholicisme et l'umbanda, avant de se convertir à l'Eglise évangélique Nova Vida à l'âge de dix-huit ans. En 1975, l'année de ses trente ans, il fonda, avec d'autres pasteurs, l'Eglise Croisade du Chemin éternel. Deux ans plus tard, en 1977, il la quitta pour créer l'Eglise Universelle du Royaume de Dieu, et demeure à ce jour son principal leader.

5 La plupart des membres de cette Eglise, comme l'ensemble des adeptes du pentecôtisme brésilien, occupent les places les plus basses de la pyramide sociale. D'après R. Mariano, "l'Universelle attire des Noirs et des mulâtres dans une proportion supérieure à la moyenne dans l'ensemble de la population. Puisque les Noirs et les mulâtres appartiennent aux couches les plus pauvres et les moins scolarisées, en raison de l'héritage de l'esclavage et de la discrimination raciale qu'ils continuent de subir dans tous les secteurs de la société brésilienne, ce fait est un autre indicateur de l'augmentation de cette dénomination religieuse, plus que les autres Eglises pentecôtistes, parmi les couches défavorisées » (Mariano, $2003: 61$ ).

6 L'Eglise Universelle adopte un modèle d'organisation institutionnelle assez centralisé et hiérarchique. Edir Macedo demeure le chef tout-puissant qui contrôle l'ensemble des activités de l'Eglise. En descendant dans la hiérarchie, on compte trois instances de pouvoir : le Conseil mondial des évêques, le Conseil des évêques du Brésil et le Conseil des pasteurs. Les temples locaux sont dirigés par des pasteurs consacrés (considérés comme étant confirmés) et des pasteurs nommés (considérés comme des auxiliaires). Pour la plupart, les pasteurs ne possèdent aucune formation en séminaires ou facultés de théologie. L'apprentissage de la fonction pastorale, à savoir prier, prêcher, exorciser, solliciter les offrandes et les dîmes, chanter, donner des conseils, etc., se fait ainsi dans la pratique. En-dessous des pasteurs, on trouve les ouvriers (obreiros), qui s'occupent du nettoyage des temples, de la réception des fidèles, du recueil des offrandes, de l'imposition des mains et des exorcismes pendant les séances de culte. Comme l'explique Mariano (2003: 57) : » Le travail dévoué de milliers et milliers d'ouvriers (obreiros) s'avère 
crucial pour le bon fonctionnement d'une dénomination religieuse qui réalise des séances de culte tous les jours de la semaine, le matin et la nuit ».

7 Les trois, quatre ou cinq séances de culte quotidiennes, réalisées dans les temples de l'Universelle, s'organisent selon des objectifs associés à chaque jour de la semaine. Ces objectifs sont appelés "chaînes". Ainsi, chaque lundi, on réalise la "chaîne de la prospérité », pendant laquelle on prie ensemble afin d'obtenir un emploi, de l'argent ou le succès dans les affaires. Les mardis sont consacrés à la «chaîne de la santé et de la désobsession ", visant à se libérer des esprits malins censés être à l'origine des maladies. Tous les mercredis, on réalise la " chaîne des Fils de Dieu ", où les fidèles prient pour tous ceux qui nécessitent d'une orientation spirituelle. Les jeudis sont consacrés à la » chaîne de la famille ", afin de ramener l'harmonie dans les foyers. Les vendredis sont les jours dédiés à la " chaîne de la libération ", pour tous ceux qui sont censés être possédés par les esprits malins, responsables pour la malchance, les vices, la pauvreté et, d'une façon générale, pour toute difficulté dans la vie quotidienne. Les samedis, on réalise la » chaîne de l'amour ", qui rassemble tous ceux qui ont des problèmes au niveau sentimental. Enfin, les dimanches sont consacrés à la "Grande rencontre avec Dieu ", quand sont célébrées des séances de culte spéciales, toujours avec la distribution de petits objets et autres biens symboliques, que les fidèles portent sur eux comme des amulettes.

Il est important de souligner que cet ordre des séances de culte quotidiennes, ainsi que l'ordre hiérarchique dont nous venons de parler, se retrouve dans tous les pays où l'Eglise Universelle s'est installée. Même dans les pays où l'Eglise ne possède que peu de temples, il est commun d'avoir au moins un évêque, qui représente la plus importante autorité religieuse de l'Eglise dans le pays. Cependant, dans les pays où l'Eglise possède un nombre plus important de temples, elle dispose de plusieurs évêques, accompagnés de pasteurs et d'ouvriers. Une des tâches des évêques à l'étranger est celle de maintenir une relation constante et même quotidienne avec le groupe dirigeant de l'Eglise, situé au Brésil, à travers de rapports d'activité, de plans d'expansion ecclésiale et de bilans financiers. Dans ce sens, bien que nous n'avions pas d'informations objectives sur ce thème, il semblerait que le contrôle international des Eglises Universelles, éparpillées de par le monde, soit fait à partir du Brésil (Rio de Janeiro et São Paulo), ainsi que des Etats-Unis. Voyons maintenant, plus en détails, comment l'Eglise est présente dans les médias.

\section{a. La présence dans les médias}

9 L'Eglise universelle est née et s'est développée en faisant usage des médias. En effet, dès l'origine, Edir Macedo loua des espaces à la Rádio Metropolitana de Rio de Janeiro, pour présenter un programme évangélique, puis a acquis au fil des années des radios émetteurs, au nombre de cinquante-deux aujourd'hui (21 AM et $31 \mathrm{FM}$ ).

10 En même temps, l'Eglise investit dans la télévision, tout d'abord en louant des espaces auprès des stations privées et plus tard, en 1989, en achetant TV Record pour 45 millions de dollars nord-américains. De nos jours, cette chaîne regroupe 63 stations de télévision et occupe la troisième place parmi les chaînes de télévision au Brésil. En outre, l'Universelle a récemment acheté la Rede Mulher (Chaîne Femme).

11 Jusqu'à ces dernières années, les programmes religieux télévisuels étaient diffusés tous les jours entre vingt-deux heures et neuf heures du matin. Aujourd'hui, ils débutent à minuit et finissent à sept heures. Mais tous les dimanches matins, l'ensemble de la Chaîne 
Record retransmet au niveau national le culte célébré au siège de l'Eglise à Rio de Janeiro ou à São Paulo.

Il est un autre domaine où dès le début l'EURD a investi, celui de la presse. Elle édite un journal hebdomadaire appelé Folha Universal, tiré à plus d'un million d'exemplaires, ainsi que les magazines Ester, Mão Amiga et Plenitude, et possède un site Internet (www.arcauniversal.com).

13 Cette Eglise accorde autant d'importance aux médias brésiliens qu'aux médias étrangers, soit en proposant à ces derniers sa propre production, soit en louant des espaces dans les médias locaux. Ainsi, selon A. Fonseca, « dès 2001, la Record est diffusée aux Etats-Unis, tandis qu'en Afrique du Sud, en Angola et au Mozambique, il est possible de suivre sa programmation dès 1998. Dans ce dernier pays, l'Universelle est propriétaire de la station Record Miramar, qui atteint quatre-vingt pour cent du territoire national, concédée par le gouvernement socialiste dans le but de contrer l'Eglise catholique » (Fonseca, $2003: 260$ ).

outre, continue l'auteur, dans la plupart des pays où elle s'est établie, l'Eglise Universelle diffuse des programmes dans les radios locales et publie des journaux: Tribuna Universal au Portugal, Universal News et Pare de Sufrir (destiné aux Hispaniques) aux Etats-Unis, Faith in Action et City News en Angleterre, Stop Suffering en Afrique du Sud, Pare de Sufrir au Chili et en Bolivie, Tribune Universelle en France.

On peut trouver plusieurs raisons à la présence dans les médias de l'Eglise Uni-verselle, comme d'ailleurs des autres Eglises pentecôtistes et néo-pentecôtistes. Une raison économique tout d'abord, en rendant publiques les dépenses ordinaires et extraordinaires de l'Eglise et en incitant les fidèles à apporter leurs contributions financières ; par prosélytisme ensuite, en donnant une grande publicité à l'Eglise, surtout par les témoignages de personnes converties et qui disent en avoir tiré bénéfice; par désir de légitimation, en montrant, aux fidèles en particulier et à la société en général, quel est son pouvoir et sa capacité d'adaptation à la modernité ; dans le but de mobiliser enfin, en faisant connaître les actions et les oeuvres de l'Eglise afin d'attirer des nouveaux fidèles (Oro, 1996).

Néanmoins, ces raisons ne possèdent pas toutes la même importance. Comme l'écrit Fonseca, «l'Universelle ne fait pas appel aux médias dans le but d'amasser de l'argent, mais plutôt pour faire la publicité de ses produits et de la sorte attirer de nouveaux fidèles. Ceux-ci vont payer les services qu'ils vont utiliser et augmenteront ainsi la file des gens payant la dîme » (Fonseca, 2003 : 278).

\section{b. Un empire économique}

17 Au Brésil, à partir des années 1990, c'est-à-dire après l'achat de Rede Record, l'EURD est au centre d'importants débats portant sur ses méthodes hétérodoxes de recueil de l'argent ainsi que sur ses investissements économiques dans différents domaines, on l'accuse de profiter pour cela des facilités fiscales prévues par la loi aux associations à but non lucratif.

18 De fait, selon R. Mariano, l'Eglise Universelle est de nos jours propriétaire au Brésil de plusieurs entreprises, surtout dans le domaine médiatique comme nous venons de le voir, auquel s'ajoute des maisons d'éditions (Editora Gráfica Universal, Universal Produções et Ediminas S/A), mais aussi une société d'enregistrement de CD (Line Records), une autre spécialisée dans le traitement de données (Uni Line), une entreprise de construction ( 
Unitec), une compagnie d'assurance (Uni Corretora), une société de production de vidéos ( Frame), une agence de voyages (New Tour), ainsi que les entreprises Unimetro Empreendimentos et Cremo Empreendimentos, parmi d'autres. La LM Consultoria Empresarial e Participação administre quelques-unes de ces entreprises.

A l'étranger, outre plusieurs radios et TV émetteurs, l'EURD possède deux institutions financières (Invest Holding et Cable Invest) dont les sièges se trouvent dans les paradis fiscaux du Royaume-Uni : les îles Caïman et Jersey (Mariano, 2003).

La plupart des fonds de l'Eglise servant à acheter ou fonder des entreprises émanent des fidèles eux-mêmes, de leurs dîmes et de leurs offrandes. La motivation de ces derniers repose sur la prédication de la Théologie de la Prospérité, théologie qui s'est épanouie aux Etats-Unis et introduite au Brésil par l'EURD. Comme le dit Mariano, »ce courant théologique, grosso modo, prône que tout chrétien [...] a acquis le droit, dans cette vie et dans ce monde-ci, à une parfaite santé physique, à la prospérité matérielle et à une vie heureuse, libérée de la souffrance et des méfaits du diable. D’après ces prédicateurs, le plus important est que Dieu non seulement a promis comme, dans le plan spirituel, il a déjà octroyé ces bénédictions à l'ensemble des croyants. Il revient alors au chrétien d'en prendre possession » (ibid.).

De cette façon, dans l'EURD, le rigorisme ascétique typique du pentecôtisme est dépassé dans une certaine mesure par l'exaltation de la richesse, par la stimulation du désir de jouir des biens matériels, ainsi que par la glorification du bien-être ici-bas. Il en découle que l'attente du Messie et de la vie éternelle est en bonne mesure remplacée par la promesse d'une récompense dans cette vie (ibid.).

Bien évidemment, l'EURD, comme d'autres Eglises néo-pentecôtistes, dans la mesure où elle a abandonné le rigorisme éthique au profit de la prospérité, a attiré à elle des individus appartenant aux couches moyennes de la société (Corten, 1995). Cependant, dans la logique de ces Eglises, la prospérité dépend de l'acte de foi du fidèle et celui-ci est mesuré à l'aune de la dîme et des offrandes qui lui sont faites. Ce point-là est d'ailleurs à l'origine d'une importante polémique suscitée par l'EURD du fait qu'elle touche un sujet où prévalent deux différentes logiques à l'œuvre au sein de la société. Tandis que pour les fidèles prédomine la logique du sacrifice et $d u$ don, qui peut être matérialisée par l'argent, pour les laïcs, payer pour une religion ne semble pas convenable (Oro et Seman, 1999). Mais, étant donné que les fidèles font des offrandes par souci de sacrifice, et qu'ils considèrent légitime et tout à fait logique le fait de dépenser leur argent pour le sacré et le surnaturel (Mariz, 1995: 28), ils ne se considèrent pas comme économiquement exploités et n'accusent pas l'Eglise d'abus financier.

\section{c. Un pouvoir politique}

23 L'EURD met en œuvre dans le champ politique le même esprit entrepreneur et rationnel qui lui a si bien réussi dans les domaines économique et médiatique. Elle obtint son premier succès au Brésil lors des élections de 1994, quand elle a fait élire six députés au Congrès national et huit députés aux assemblées des Etats, chiffres portés respectivement à dix-sept (quatorze d'entre eux venant de l'Eglise elle-même et trois autres étant appuyés par l'Eglise) et vingt-six en 1998 (Fonseca, 1998 : 20). La participation de l'EURD à la politique brésilienne a fortement contribué à former le plus grand bloc évangélique ( bancada evangélica) de l'histoire du Brésil, avec cinquante-trois députés appartenant à treize Eglises différentes (Magazine Época, 2 octobre 2000). Il s'agit en effet du « quatrième 
plus grand bloc capable de faire barrage lors de votes et de provoquer des négociations avec le gouvernement, comme d'ailleurs il a déjà fait » (Diário Catarinense, Florianópolis, 30 juillet 2000).

Une étude effectuée par P. Freston a montré que des quatorze députés de l'EURD, membres de cette Eglise depuis quinze ans environ, quatre-vingt pour cent étaient des pasteurs ou des évêques, ayant développé des activités dans les médias, et aucun, à une exception près, n'était inscrit à un parti politique avant son élection. Freston présente aussi le profil type du député de l'EURD :» [...] is thus a White man in his 40s, without higher education; a non-Protestant until converting to the Universal Church in the 1980s, he is now a pastor and presenter of church radio or television programs, and possibly involved in church social programs; he is now in his first legislature, with no previous political experience or party involvement " (Freston, 2000 : 300).

Les dix-sept députés de l'EURD ont tous été élus dans des partis de centre-droit. Néanmoins, s'ils ont soutenu l'ancien président Fernando Henrique Cardoso lors de son premier mandat, ce ne fut pas le cas lors du second mandat. D'après le discours de l'Eglise, le passage à l'opposition est dû à la faible attention donnée par le gouvernement aux problèmes sociaux.

Pour obtenir un tel succès dans le champ politique, l'EURD réalise des œuvres d'assistance sociale - ce qui lui permet, comme remarque A. Corten (1997: 29), « [...] de se constituer une base électorale ». De plus, elle mobilise sa machine médiatique, faisant usage, dans le champ politique et dans la construction de sa représentation politique, de quelques principes axiologiques et doctrinaires de l'Eglise. Ainsi, elle actionne le principe millénariste, selon les nouvelles véhiculées par la presse nationale sur la corruption dans le domaine politique. Bien qu'elle ne soit pas seule à le faire, l'EURD s'indigne des scandales de corruption politique et en appelle à « une grande réforme spirituelle dans la vie du peuple brésilien", par la bouche de son principal leader politique, l'évêque Rodrigues. Pour elle, la lutte pour la moralisation de la politique suppose la conception d'une bataille spirituelle fondée sur un discours qui fait directement appel aux « forces invisibles " à l'œuvre dans la politique (Corten et Mary, 2000), c'est-à-dire que la symbolique de la diabolisation est une clef essentielle pour analyser les «maux » de la politique dus à Satan:» Les esprits qui agissent dans la politique, a dit récemment l'évêque Rodrigues, ce sont les esprits dominateurs, les princes des ténèbres " (Journal do Brasil, Rio de Janeiro, 29 octobre 2001). L'EURD est sûre de pouvoir libérer la politique de l'emprise du pouvoir démoniaque en instaurant la politique du Bien contre la politique du Mal.

Il faut souligner que cette mainmise politique de l'EURD ne concerne que le Brésil, car des tentatives semblables effectuées au Portugal se sont soldées par un échec.

\section{La transnationalisation de l'Église Universelle du Royaume de Dieu}

Venons à présent à un autre aspect du succès rencontré par l'EURD : son implantation dans quatre-vingt pays. Cet exploit est d'autant plus impressionnant quand on sait qu'à l'étranger la gestion du pouvoir au sein de cette Eglise est encore plus centralisée et que les pasteurs sont pour la plupart des Brésiliens. D'ailleurs, le recrutement des personnels locaux ne concerne que deux niveaux de l'organisation de l'Eglise: d'une part, le 
recrutement d'«ouvriers» (obreiros), pour la plupart des jeunes des deux sexes, qui reçoivent les fidèles et surveillent leur comportement pendant le culte; d'autre part, un groupe formé de différents spécialistes, tels que les avocats, qui ouvrent dans l'intérêt de l'Eglise et sont des intermédiaires avec les autorités des différents pays.

Comme le concept de transnationalisation - de même que ceux très proches de globalisation, mondialisation, déterritorialisation, etc. - est controversé et polysémique ${ }^{4}$, je précise que c'est dans le sens que lui donnent B. Badie et M. C. Smouts que j'utilise ce terme. Selon ces auteurs, on assiste de nos jours à la montée de flux transnationaux relevant de groupes privés qui se dotent de moyens d'actions adaptés et qui se réalisent hors de l'Etat et en ignorant les frontières. Pour ces auteurs, une relation transnationale est «toute relation qui, par volonté délibérée ou par destination, se construit dans l'espace mondial au-delà du cadre étatique national et qui se réalise en échappant au moins partiellement au contrôle ou à l'action médiatrice des Etats » (Badie et Smouts, 1995: 70). Ils distinguent également les flux produits par des organisations non gouvernementales et ceux qui dérivent de la combinaison d'actions individuelles multiples. Les flux religieux, organisés par les Eglises, les associations culturelles, les sectes ou par les groupes de prêcheurs, se rattachent à la première catégorie. La seconde est constituée par l'agrégation de démarches individuelles (ibid. : 71).

L'expansion internationale de l'Eglise Universelle découle de sa seule décision en fonction de ses propres intérêts et les rapports qu'elle entretient avec les Etats se situent uniquement sur le plan légal, pour obtenir passeports et visas pour ses pasteurs, ou payer ses impôts. Le choix d'un pays ou d'une ville où s'installer obéit toujours à la même procédure. Le groupe directeur établit en premier lieu une liste des pays et des villes où l'Eglise est susceptible de s'installer, en tenant en compte la présence de Brésiliens ou d'Hispaniques qui sont autant de membres potentiels. Une fois le choix effectué, des locaux sont loués, surtout des cinémas ou des commerces désactivés dans des zones de grande fréquentation. C'est alors que le travail de prosélytisme peut commencer.

31 L'expansion internationale de l'Eglise Universelle a débuté en 1985 avec l'ouverture d'un temple au Paraguay. Puis ce sont les Etats-Unis en 1986 et l'Argentine et le Portugal en 1989 (Freston, 2001). Si au début l'expansion est lente, le rythme s'accélère à partir des années 1990 : 221 temples en 1995, 500 en 1998 et 1.000 en 2001. Et la croissance continue. Selon A. Corten, l'EURD est présente de nos jours dans tous les pays d'Amérique latine, sauf en Haïti, dans la moitié de l'Afrique, au Canada, aux Etats-Unis, et dans une douzaine de pays européens. Elle se développe aussi en Europe de l'Est et dans quelques pays d'Asie (Corten, 2002). Soit au total quatre-vingts pays, même si dans la plupart d'entre eux la présence de l'Eglise n'est que symbolique. En effet, les pays où l'on compte plus de 50 temples sont, outre bien sûr le Brésil, l'Argentine, le Venezuela, le Portugal, le RoyaumeUni, la Côte d'Ivoire, le Mozambique, l'Afrique du Sud et les Etats-Unis.

Examinons maintenant trois pays - Argentine, Portugal et Afrique du Sud - où l'Eglise Universelle connaît une grande expansion au niveau quantitatif et où elle a su s'adapter aux cultures locales. Nous les avons choisis parce que, outre fournir des exemples issus de différents continents, nous disposons à présent de données ethnographiques consistantes sur ces pays qui, d'une certaine façon, constituent trois»cas exemplaires» de l'adaptation de cette Eglise aux cultures locales. Il s'agit là, probablement, d'une situation récurrente dans d'autres pays où l'EURD a connu du » succès » dans sa multiplication de temples et dans le rassemblement de fidèles. Cela pourrait nous amener à penser que la difficulté à trouver des brèches pour l'insertion dans les cultures locales puisse être un 
élément d'explication à « l'échec » relatif de l'Eglise dans d'autres pays, où elle stagne ou avance lentement. Toutefois, seulement de nouvelles recherches dans les différents pays pourront confirmer une telle hypothèse.

\section{a. L'Eglise Universelle du Royaume de Dieu en Argentine}

33 C'est à la fin des années 1980 que l'EURD ouvre ses premiers temples en Argentine, à Buenos-Aires. Si les débuts sont difficiles, à partir de 1995, elle connaît un grand développement. Aujourd'hui, on compte plus de quatre-vingts temples répartis dans les principales villes du pays.

Le discours de l'EURD est bien reçu, en particulier parmi les segments supérieurs des basses couches sociales et les segments inférieurs des couches moyennes urbaines. Il s'agit des secteurs qui, comme au Brésil, souffrent à l'heure actuelle des effets récessifs de la politique néolibérale et qui espèrent pouvoir, grâce à l'Eglise, surmonter la crise socioéconomique et trouver le bien-être qui leur fait défaut au quotidien (Oro et Seman, 2001).

Bien qu'il soit difficile d'avancer un chiffre quant au nombre de fidèles en Argentine, $P$. Seman estime pouvoir l'évaluer à partir de la capacité de mobilisation sociale de l'EURD. Il signale qu'à Noël 2001, elle a rassemblé entre 40.000 et 50.000 personnes dans un stade de football de Buenos Aires (Seman, 2003).

De même qu'au Brésil, en Argentine l'EURD est présente dans les médias, notamment à la radio et à la télévision avec des programmes nocturnes, et par le journal qu'elle publie, Folha Universal. Aussi elle mène des campagnes d'assistance sociale dans le but d'acquérir une plus grande légitimité, surtout dans les départements et les villes de province où elle a dû faire face à une certaine hostilité en raison de son prosélytisme dynamique.

Le relatif succès de l'Eglise Universelle en Argentine est dû à son adaptation à la culture locale. En effet, l'un des aspects importants de la doctrine de l'EURD est la représentation du démon comme étant à l'origine des maux et de toutes les afflictions. Au Brésil, cette diabolisation prend le visage des religions afro-brésiliennes (candomblé, umbanda, quimbanda). En Argentine aussi, la figure du démon est présente dans les sensibilités religieuses, en particulier du catholicisme populaire, bien qu'elle demeure dans l'ombre. Néanmoins, étant donné que dans la société argentine les religions afro-brésiliennes n'ont pas le même impact qu'au Brésil, la diabolisation affecte surtout les guérisseurs ( curanderos), ou relève du mauvais-œil et de la jalousie. Mais - et ils montrent là leur grande capacité d'adaptation - les pasteurs ajustent leurs exorcismes » aux aspects psychologisés de la culture des fidèles argentins»(Seman et Moreira, 1998: 103). Autrement dit, dans ce pays, les démons ne sont pas identifiés, comme au Brésil, avec les entités du panthéon afro-brésilien mais plutôt avec le mal-être, entendu dans une perspective psychologique. Ainsi, l'Eglise Universelle ne renonce pas à la diabolisation, mais elle la réinterprète en mettant en rapport certains éléments propres de la société argentine avec son discours peuplé d'entités malignes: «Les fidèles apprennent que les démons sont à l'origine du mal-être qu'auparavant ils expliquaient de façon différente et l'Eglise modifie le répertoire des infortunes censés être causés par des démons, ainsi que la manière dont ils se produisent » (ibid. : 104).

L'EURD offre donc aux fidèles des couches populaires argentines ce qu'elles ne trouvent pas dans les différentes versions du pentecôtisme local, en attribuant au démon les maux 
de la vie urbaine et moderne tels que l'angoisse existentielle, le stress et la dépression, maux dont elle déclare pouvoir libérer les fidèles moyennant l'exorcisme.

Cela étant, pour les adeptes argentins de l'EURD, celle-ci ne constitue pas seulement une Eglise et des fidèles de plus, mais elle correspond à une façon d'être pentecôtiste spécifique et nouvelle, adaptée au contexte argentin.

\section{b. L'Eglise Universelle du Royaume de Dieu au Portugal}

40 L'implantation de l'Eglise Universelle au Portugal s'est faite fin 1989, aux environs de Lisbonne, concrétisant ainsi une étape importante de la stratégie d'expansion de l'EURD vers d'autres pays européens et africains de langue portugaise (Freston, 2001). Ceci, comme le dit Marion Aubrée, parce qu'il existe » [...] des migrants d'origine portugaise dans presque toutes les capitales d'Europe de l'Ouest et c'est à travers cette filière migratoire que l'EURD a commencé à se développer dans cette région du monde» (Aubrée, 2000 : 155).

41 L'implantation au Portugal a été plus facile que dans beaucoup d'autres pays, principalement pour trois raisons : l'absence de la barrière de la langue pour les pasteurs brésiliens; l'absence de la barrière religieuse étant donné que, comme au Brésil, la plupart de la population portugaise est catholique; l'entrée commune dans l'économie néolibérale à partir de la décennie 1970 (Mafra, 2003).

Néanmoins, lors de son arrivée au Portugal, l'EURD fut prise à partie lors de débats locaux relatifs aux sectes et à leurs méfaits. Plus tard, la presse portugaise reproduisit des informations venues du Brésil concernant les scandales financiers et les profits de l'Eglise. En 1996, les journaux annoncèrent que l'EURD faisait l'objet d'une enquête judiciaire. Et en 1999, l'EURD revint à la une des journaux dans le cadre des débats sur la reformulation de la loi de Liberté religieuse, octroyée cette année-là. Mais c'est l'année 1995 qui fut la plus difficile pour l'EURD au Portugal, car elle eut à subir des actions xénophobes après avoir déclaré son intention d'acheter le Coliseu do Porto et de construire un parti politique, le Parti des Gens (Partido da Gente).

Le Coliseu est vu par les habitants de Porto comme étant le plus traditionnel parmi les lieux de spectacles et quand, en août 1995, l'UAP (l'une des principales compagnies d'assurance de la région), son propriétaire, a annoncé que l'EURD était en train d'acheter l'immeuble, le syndicat des artistes, des intellectuels, des politiciens et une partie de la population, ont exprimé publiquement leur mécontentement. Trois mois plus tard, une manifestation publique qui se tenait devant le Coliseu fut retransmise par la télévision. C'est alors que furent tenus des propos xénophobes, bien que la teneur générale des discours portait sur la défense de l'immeuble, » symbole de la vie publique de Porto ». Une semaine plus tard le président lui-même, Mario Soares, intervint dans le conflit et, finalement, en janvier 1996, la » Société des Amis du Coliseu do Porto ", qui venait d'être créée, a acheté l'immeuble. De même, le projet de création d'un parti politique n'eut pas de suite en raison de l'opposition manifestée par plusieurs secteurs de la société portugaise. Ce projet de l'Universelle a ainsi échoué de la même manière que l'acquisition du Coliseu do Porto (Mafra, 2002).

Quoi qu'il en soit, et comme nous l'avons dit, ce fut la seule tentative de l'Eglise Universelle de participer directement à la vie politique d'un pays en dehors du Brésil. Ce qui ne veut pas dire pour autant que cette Eglise ne soit pas impliquée et insérée dans 
l'espace public des pays où elle s'est installée, notamment par sa présence dans les médias et son travail d'assistance sociale.

Néanmoins si, au Portugal, l'EURD n'a pu faire aboutir les deux projets dont nous venons de parler, elle a, comme le souligne $\mathrm{C}$. Mafra, recueilli les fruits de sa persévérance, grâce surtout à l'évêque Marcelo Brayner, alors responsable de l'Eglise au Portugal, qui a su» [...] renforcer les aspects plus libertaires de l'influence de l'EURD, a défendu fermement le droit des citoyens à avoir des opinions religieuses diffé-rentes, le droit à la liberté et à l'égalité devant la loi, et qui a su faire preuve de son mépris à l'égard de toute persécution. Ce faisant, il a mobilisé l'opinion publique portugaise en sa faveur et il a indiqué une voie possible pour la solidification de l'institution au Portugal, tout en menant le combat public rigoureusement, s'appuyant sur la loi »(Mafra, 2002 : 204).

D'ailleurs, remarque Mafra, tant au Brésil qu'au Portugal l'EURD a toujours su utiliser en sa faveur les » théories persécutives ", en même temps qu'elle a prêté sa contribution à la construction du pluralisme religieux au Portugal (ibid.: 63-65). Malgré donc la mobilisation des médias portugais et les différentes oppositions au sein de la société, l'EURD n'a pas perdu sa popularité au Portugal. Le démontre le fait «qu'en 1994 elle possédait treize temples et qu'en 1999 elle en avait plus de quatre-vingt-dix. Ceci veut dire que, comme au Brésil, les conflits spectaculaires entre la presse et l'Eglise semblent avoir provoqué un résultat contraire à celui qui était recherché : depuis 1995 [...] l'Eglise grandit énormément » (Mafra, 2001:2).

En outre, et à nouveau comme au Brésil, depuis son installation au Portugal, l'EURD s'est attachée à structurer son réseau médiatique, en achetant des radio-émetteurs qui étaient dans une situation économiquement difficile, en diffusant des programmes télévisés et le journal Tribuna Universal. Ainsi, l'Eglise a pris sa place dans la vie publique portugaise, faisant connaître ses activités religieuses et ses campagnes d'assistance sociale ${ }^{5}$.

Comme dans les autres pays, il est difficile d'estimer le nombre de fidèles de l'EURD au Portugal. Selon Mafra, depuis 1994, les évêques et les pasteurs de cette Eglise annoncent le chiffre - contestable - de plus de 200.000 fidèles. Le profil social des adeptes de l'Eglise au Portugal ne serait pas différent de celui du Brésil : il s'agit surtout de femmes, issues des couches populaires et ayant une faible scolarité, bien qu'elle attire aussi un public de classe moyenne et même moyenne haute (Mafra, 2002b).

Clara Mafra attire l'attention sur deux aspects importants pour la compréhension du développement de l'EURD dans ce pays. Tout d'abord, dès le début, les pasteurs brésiliens ont tenu un discours où ils soulignaient le besoin de combattre le démon au Portugal, [...] un pays qui, disaient-ils, vivait dans la 'queue de la communauté européenne alors que sa place est à la tête'; qui avait des gouverneurs peu préoccupés par les problèmes internes; qui comptait sur des politiciens n'accordant que peu d'attention à la situation des personnes âgées, des prisonniers, des enfants, des chômeurs, des gens faiblement scolarisés » (Mafra, 2003: 170).

Paul Freston remarque aussi que l'implantation de l'EURD au Portugal coïncide avec l'entrée de ce pays dans la Communauté économique européenne, et que ce fait a conduit des gens, qui ne voyaient pas leurs aspirations suscitées par la nouvelle condition du pays se concrétiser, à adhérer à cette Eglise, attirés par sa Théologie de la Prospérité (Freston, 2001).

Deuxièmement, l'EURD a su s'adapter à la culture portugaise, comme le démontre l'exemple de la » société de quartier» (sociedade de bairro), selon l'expression d'Antonio 
Firmino da Costa. Dans cette société, la territorialité est fondamentale ; les quartiers sont toujours dépendant d'un centre et sont les uns les autres en concurrence pour la plus grande » authenticité ». L'Eglise Universelle n'est pas entrée dans une telle société, où d'ailleurs le neuf et l'étranger sont marginalisés. Elle s'est lentement appropriée la façon de faire des quartiers où les associations locales font office de médiateurs entre la population et les mairies. L'EURD a mis en place un réseau territorialisé, composé d'environ cent lieux de culte, avec Lisbonne comme siège central et plus précisément l'Eglise de l'Empire (Igreja do Império). Ainsi, le réseau institutionnel de l'EURD fonctionne de façon semblable à une société de quartier. Comme le remarque Mafra, » les Eglises sont également rivales au niveau national» (Mafra, 2001: 11). Mais, elles diffèrent des associations de quartier en ce qu'elles ne concourent pas pour la fête de saint la » plus authentique ou la plus grandiose ", mais plutôt pour " [...] la fête d'anniversaire de l'Eglise la plus fastueuse, pour le temple qui a réussi à faire venir pour sa célébration locale l'homme de Dieu le plus prestigieux, pour le plus grand nombre de baptêmes célébrés dans le mois, pour la plus importante quantité de dons rassemblés lors de la dernière campagne » (Mafra, $2001: 12$ ).

Il est évident que l'EURD a recours à ce langage, qui n'est non pas le sien mais celui de la société de quartier, pour en faire un » langage de séduction », un » [...] appât, un appel d'où la cosmologie de l'EURD va gagner en crédibilité » (ibid.), en même temps qu'un espace d'expression pour tous ceux qui ne sont pas satisfaits de cette dynamique identitaire. Cela étant, »l'Eglise Universelle a mis en œuvre au Portugal une stratégie dont on peut dire qu'elle substitue la compétition de la fête du saint par la fête de l'Eglise » (Mafra, 2003: 172).

Enfin, Mafra reconnaît l'importance des pasteurs brésiliens de l'Eglise Universelle pour trouver les points de convergence entre "plusieurs théories implicites dans le sens commun portugais et les préceptes normatifs et doctrinaires de l'Eglise ». Le prestige de ces pasteurs-là émane du fait de pouvoir » [...] avec leurs discours chargés de métaphores et des termes polysémiques, indiquer à leurs adeptes comment ils pourront recréer des totalisations provisoires [adaptées] au point de vue local » (Mafra, $2001: 20$ ).

\section{c. L'Eglise Universelle du Royaume de Dieu en Afrique du Sud}

54 L'Eglise Universelle arrive en Afrique du Sud en 1993 en partie avec les émigrés angolais et mozambicains. Mais, comme le dit Corten, dès le début, elle cherche à être reconnue autrement que comme une Eglise d'immigrés. »Avec dix-sept temples en 1995 (principalement dans la région de Johannesburg), l'EURD s'est implantée dans toutes les provinces et comptait 115 temples en 1998 et 181 en 2001 (Corten, $2003: 138$ ). En dépit de ce nombre important, elle est maintenue à l'écart des canaux télévisuels par l'Etat, mais a accès à la radio et à la presse par un journal hebdomadaire, le Universal News, tiré à cent mille exemplaires.

Corten rappelle que l'EURD s'est implantée en Afrique du Sud juste avant que la page de l'apartheid soit tournée. En effet, c'est en 1990 que Mandela a été libéré et en 1994 qu'ont eu lieu les premières élections libres. Les Eglises pentecôtistes ont joué un rôle ambigu dans la lutte contre l'apartheid. La plupart d'entre elles » avaient plutôt tendance à suivre la ligne de la très afrikaner NGK (Eglise réformée hollandaise) ayant prétendu trouver dans la Bible un fondement à l'apartheid, que d'adopter les positions plus progressistes des Eglises méthodiste, anglicane et catholique » (Corten, $2003: 140)$. 

l'apartheid, l'EURD est arrivée à point nommé pour occuper une place laissée vacante. Il faut dire tout d'abord que, selon une lecture sud-africaine, l'EURD est une Eglise provenant d'un pays dit d'intégration raciale, de métissage réussi, autrement dit, un pays de conciliation inter-raciale, tel que l'envisageait l'Afrique du Sud de l'après-apartheid. Néanmoins, la fin de l'apartheid politique ne signifiait pas la fin de l'apartheid économique et social. C'est là que l'EURD, ainsi que d'autres Eglises qui prêchent la Théologie de la Prospérité, sont intervenues. Mais, alors que ces dernières attirent plutôt les couches moyennes, l'EURD s'adresse davantage aux couches populaires. En effet, » l'Eglise Universelle est de nature à offrir aux populations frustrées par une amélioration des conditions de vie qui se fait attendre, un canal fantasmatique d'attente. L'esprit de défi et de risque, enseigné à l'Universelle, renforce la confiance en soi-même. [...] Premier signe de succès social, encouragé dans les prédications » (Corten, 2003 : 143).

Par ailleurs, à l'heure actuelle, le pouvoir politique sud-africain défend l'idéologie de la renaissance africaine, conçue comme un projet de modernisation visant à donner à l'Afrique du Sud une nouvelle place dans l'économie mondiale. Or, selon Corten, »l'EURD trouve dans cette idéologie de la Renaissance africaine et du rôle que l'Afrique du Sud s'y attribue, une conception homologue à sa conception de l'uni-versalité. Cette homologie [...] se double, dans la phase actuelle, d'une position assez symétrique vis-à-vis du néolibéralisme. Le modèle de croissance de l'EURD relève d'une stratégie de multinationale, s'implantant ici ou fermant là en fonction d'études de marché » (ibid.).

Il apparait donc que le succès relatif de l'implantation de l'EURD en Afrique du Sud tient au fait que cette Eglise a su s'adapter à l'actuel contexte politico-culturel de ce pays. L'EURD » semble avoir découvert un rôle spécifique à jouer dans le contexte postapartheid en offrant certains imaginaires de mobilité sociale et en s'associant au rôle de Renaissance africaine [...]» (ibid.).

\section{Conclusions}

L'Eglise Universelle est née au Brésil et s'est répandue de par le monde parallèlement à la dite globalisation. Il est possible que cette situation ait contribué à favoriser la transnationalisation de cette Eglise. Néanmoins, j'ai montré dans cet article que son universalisation est due avant tout à sa capacité à s'adapter aux cultures locales. C'est pourquoi je considère que la transnationalisation de l'EURD est un succès, qui n'est pas dû au hasard mais qui découle de ses activités et de ses pratiques cohérentes et logiques.

60

J'ai laissé de côté l'analyse des changements locaux entamés par l'Eglise Univer-selle ${ }^{6}$. De même demeurent ouvertes deux questions théoriques, que je vais ici à peine esquisser. En premier lieu, en se transnationalisant, cette Eglise a-t-elle changé et, si oui, en quoi? A ce stade de son expansion, il me semble qu'elle a réussi à s'adapter aux cultures locales tout en préservant son propre ethos, c'est-à-dire qu'elle est parvenue, malgré les changements évidents qui accompagnent tout processus de transnationalisation, à préserver ses principes fondamentaux qui font d'elle une Eglise à part entière dans le champ pentecôtiste latino-américain.

61 En deuxième lieu, comment cette Eglise réussit-elle à articuler visées universelles et visées locales? C. Mafra propose une réponse à laquelle j'adhère puisqu'elle correspond à l'analyse que j'ai faite de l'implantation de l'EURD en Argentine, au Portugal et en Afrique 
du Sud. Selon elle, l'Eglise Universelle parvient à maintenir un équilibre entre les pressions locales et les expectatives modernisantes transnationales, en n'ignorant aucun des deux côtés et sans que les gens soient obligés de choisir l'un des deux (Mafra, 2002 : 113).

\section{BIBLIOGRAPHIE}

AUBRÉE, Marion

2000, « La diffusion du pentecôtisme brésilien en France et en Europe : le cas de l'I.U.R.D. » in Lerat, Christian et Rigal-Cellard, B. (éds.), Les mutations transatlantiques des religions, Bordeaux : PUB, pp. 149-157.

2001, « Dynamiques comparées de l'Eglise Universelle du Royaume de Dieu au Brésil et à l'étranger », in Bastian, J. P., Champion, F. et Rousselet, K. (éds.), La globalisation du religieux, Paris : L'Harmattan, pp. 113-124.

BADIE, Bertrand et SMOUTS, M. C., 1992, Le retournement du monde. Sociologie de la scène internationale, Paris : Presse de la Fondation nationale des sciences politiques \& Dalloz.

CAPONE, Stefania, 2000, «'Du pareil au même': olhares críticos da antropologia francesa sobre as noções de globalização, transnacionalização e postmodernismo ", Communication présentée à Gramado, Brésil, lors de la 23 ${ }^{\text {ème }}$ Reunião Brasileira de Antropologia, 16-19 juin.

CORTEN, André

1995, Le pentecôtisme au Brésil, Paris : Karthala.

1997, « Pentecôtisme et politique en Amérique latine », Problèmes d'Amérique Latine, 24, janviermars, pp. 17-32.

1999, « Pentecôtisme et 'néo-pentecôtisme' au Brésil », Archives des sciences sociales des religions, 105(1), janvier-mars, pp. 163-183.

2002, «A Igreja Universal : uma máquina multinacional que reponde às novas necessidades religiosas ", Petrópolis, Revista de Cultura Vozes, pp. 38-47.

2003, « A Igreja Universal na Africa do Sul », in Oro, Ari Pedro ; Corten, André et Dozon, JeanPierre (éds.), Igreja Universal do Reino de Deus : do Rio para o mundo, São Paulo : Editora Paulinas, pp. 137-145.

CORTEN, André et MARY, André (éds)., 2000, Imaginaires politiques et pentecôtismes. Afrique/Amérique latine, Paris : Karthala.

FONSECA, Alexandre Brasil

1998, « A maior bancada evangélica », Tempo e Presença, São Paulo, Cedi, 302, novembre/ décembre, pp. 20-23.

2003, « Igreja Universal do Reino de Deus : um império midiático » in Oro, Ari Pedro ; Corten, André et Dozon, Jean-Pierre (éds.), Igreja Universal do Reino de Deus : do Rio para o mundo, São Paulo : Editora Paulinas, pp. 259-280. 
FRESTON, Paul

2000, «The Political Evolution of Brazilian Pentecostalism : 1986-2000 » in Corten, André et Mary, André (éds.), Imaginaires politiques et pentecôtismes : Afrique/ Amérique latine, Paris : Ed. Karthala, pp. 287-306.

2001, «The transnationalization of Brazilian Pentecostalism. The Universal Church of the Kingdom of God » in Corten, André, Marshall-Fratani, Ruth (éds.), Between Babel and Pentecostalism. Transnational Pentecostalism in Africa and Latin America, Londres : Hurst \& Company, pp. 196-215.

MAFRA, Clara

2001, « Sobre a qualidade da mediação institucional da Igreja Universal do Reino de Deus (IURD) com foco no caso de Portugal ", communication présentée à la Conférénce de la SISR à Ixtapan de la Sal (Mexique), 20-24 août.

2002, Na posse da palavra. Religião, conversão e liberdade pessoal em dois contextos nacionais, Lisboa : Instituto de Ciências Sociais da Universidade de Lisboa.

2003, « A Igreja Universal do Reino de Deus em Portugal », in Oro, Ari Pedro ; Corten, André et Dozon, Jean-Pierre (éds.), Igreja Universal do Reino de Deus : do Rio para o mundo, São Paulo : Editora Paulinas, pp. 165-176.

MARIANO, Ricardo

1999, Neopentecostais: sociologia do novo pentecostalismo no Brasil, São Paulo : Edições Loyola.

2003, « Igreja Universal do Reino de Deus : um império econômico », in Oro, Ari Pedro ; Corten, André et Dozon, Jean-Pierre (éds.), Igreja Universal do Reino de Deus : do Rio para o mundo, São Paulo : Editora Paulinas, pp. 53-67.

MARIZ, Cecília, 1995, «El debate en torno del Pentecostalismo Autônomo en Brasil », Sociedad y Religión, Buenos Aires, 13, pp. 21-32.

ORO, Ari Pedro, 1996, Avanço pentecostal e reação católica, Petrópolis : Editora Vozes.

ORO, Ari Pedro, CORTEN, André et DOZON, Jean-Pierre (éds.), 2003, Igreja Universal do Reino de Deus : do Rio para o mundo, São Paulo : Editora Paulinas.

ORO, Ari Pedro et SEMAN, Pablo

1999, « Neopentecostalismo e conflitos éticos », Religião e Sociedade, Rio de Janeiro, vol. 20, 1, pp. 39-54.

2001, « Brazilian Pentecostalism Crosses National Borders », in Corten, André et Marshall-Fratani, Ruth (éds.), Between Babel and Pentecostalism. Transnational Pentecostalism in Africa and Latin America, Londres : Hurst \& Company, pp. 181-195.

PIERUCCI, Antonio Flavio et PRANDI, Reginaldo, 1996, A realidade social das religiões no Brasil, São Paulo : Hucitec.

RUUTH, Anders et RODRIGUES, Donizete, 2000, « A Kingdom of Heaven in Expansion - Until When? Some Considerations about the Universal Church of the Kingdom of God in Brazil », in Rodrigues, Donizete et Del Rio, Pablo, The Religious Phenomenon. An Inter-disciplinary Approach, Madrid : Fundación Infancia y Aprendizaje, pp. 29-42.

SANCHIS, Pierre, 1997, « Pentecostalismo e Cultura Brasileira », Religião e Sociedade, Rio de Janeiro, 18(2), pp. 123-126. 
SEGATO, Rita, 1997, « Formações de diversidade : nação e opções religiosas no contexto da globalização », in Oro, Ari Pedro et Steil, Carlos Alberto (éds.), Globalização e Religião, Petrópolis : Editora Vozes, pp. 219-248.

SEMAN, Pablo, 2003, «A Igreja Universal do Reino de Deus na Argentina », in Oro, Ari Pedro ; Corten, André et Dozon, Jean-Pierre (éds.), Igreja Universal do Reino de Deus : do Rio para o mundo, São Paulo : Editora Paulinas, pp. 69-91.

SEMAN, Pablo et MOREIRA, Patrícia, 1998, «La Iglesia Universal del Reino de Dios en Buenos Aires y la recreación del diablo a través del realineamiento de marcos interpretativos ", Sociedad y Religión, Buenos Aires, 16-17, pp. 95-110.

WILLAIME, Jean-Paul, 1999, « Le Pentecôtisme: contours et paradoxes d'un protestantisme émotionnel », Archives des sciences sociales des religions, 105(1), janvier-mars, pp. 5-28.

\section{NOTES}

1. Selon ces paramètres les deux premières Eglises sont elles aussi pentecôtistes, à savoir l'Assemblée de Dieu, fondée en 1911, avec 4,5 millions de fidèles, 22.000 temples et 21.000 pasteurs, et l'Eglise Congrégation Chrétienne du Brésil, fondée en 1910, avec 2,2 millions de fidèles, 14.300 temples et 18.700 pasteurs. L'EURD, en raison des changements introduits dans le pentecôtisme traditionnel, aussi bien par rapport à ses activités sociales qu'à sa doctrine, est considérée comme une Eglise néo-pentecôtiste. Ce dernier point, cependant, est loin de faire l'unanimité parmi les spécialistes (Oro, 1996 ; Pierucci et Prandi, 1996 ; Mariano, 1999 ; Willaime, 1999 ; Corten, 1999 ; Aubrée, 2001). D’après Paul Freston, «le Brésil détient la deuxième plus grande communauté de pratiquants du protestantisme au monde, et la plus grande communauté de pentecôtistes » (Freston, $2001:$ 198).

2. Ce fait semble être courant dans la mouvance pentecôtiste. A ce propos, P. Sanchis (1997) montre de manière exemplaire comment le pentecôtisme s'est intégré, de façon toujours plus organique, dans le champ culturel et religieux du Brésil, et notamment du Brésil populaire.

3. Le terme carioca désigne les habitants de la ville de Rio de Janeiro.

4. Pour une analyse des controverses autour de ces concepts dans l'anthropologie française, voir Capone, 2002.

5. Selon Freston, au Portugal, l'EURD met l'accent sur le travail social : distribution de nourriture et de vêtements, travail avec les drogués, avec les personnes âgées et avec les orphelins. De cette façon, elle cherche une légitimité niée par des importants secteurs sociaux de ce pays (Freston, 2001: 203-204).

6. Ainsi, par exemple, selon Clara Mafra, au Portugal l'EURD interfère dans les coutumes traditionnelles de la population, telles que le port du noir par les veufs, les fêtes des villages, les touradas, les bougies pour les morts, la réalisation du paiement des promesses, etc. (Mafra, 2002 : 84). 


\section{RÉSUMÉS}

Ce texte analyse la dimension transnationale de l'Eglise Universelle du Royaume de Dieu, une Eglise pentecôtiste brésilienne née en 1977 à Rio de Janeiro et qui jouit aujourd'hui d'une grande visibilité au Brésil et dans d'autres pays en raison de son insertion dans les médias, l'économie et la politique. Nous verrons que son implantation dans quatre-vingts pays est due à sa grande capacité à s'adapter aux différentes cultures locales. Dans ce sens seront analysés trois cas, à savoir l'implantation de cette Eglise en Argentine, au Portugal et en Afrique du Sud.

This paper analyses the transnational dimension of the Universal Church of the Kingdom of God, a Brazilian Pentecostal church founded in Rio de Janeiro in 1977. It enjoys today a large visibility in Brazil as well as in other countries because it is well introduced in the media, the economy and the politics. We shall see that its introduction in 80 different countries was possible thanks to its great capacity to adapt to different local cultures, as shown by three different case studies, i.e. the introduction of this Church in Argentina, in Portugal and in South Africa.

\section{INDEX}

Keywords : globalisation, transnationalization, pentecostalism, Universal Church of the Kingdom of God

Mots-clés : mondialisation, transnationalisation, pentecôtisme, Eglise Universelle du Royaume de Dieu

\section{AUTEUR}

\section{ARI PEDRO ORO}

Ari Pedro Oro est professeur d'anthropologie à l'Université fédérale de Rio Grande do Sul (Brésil) et chercheur du CNPq. Il mène actuellement des recherches sur latransnationalisation des religions afro-brésiliennes et des Eglises pentecôtistes « brésiliennes » dans les pays du Mercosul, et notamment en Uruguay et Argentine. Il est l'auteur de plusieurs ouvrages, parmi lesquels Axé Mercosul : a expansão das religiões afro-brasileiras para os países do Prata (Ed. Vozes, 1999), et éditeur de l'ouvrage collectif Globalização e Religião (Ed. Vozes, 1997). 